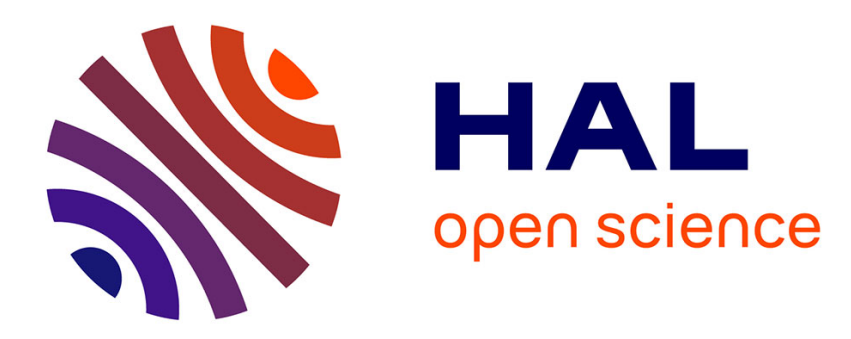

\title{
Advances in optically pumped He4 magnetometers: Resonance and nonresonance techniques
}

\author{
R.E. Slocum
}

\section{To cite this version:}

R.E. Slocum. Advances in optically pumped He4 magnetometers: Resonance and nonresonance techniques. Revue de Physique Appliquée, 1970, 5 (1), pp.109-112. 10.1051/rphysap:0197000501010900 . jpa-00243341

\section{HAL Id: jpa-00243341 https://hal.science/jpa-00243341}

Submitted on 1 Jan 1970

HAL is a multi-disciplinary open access archive for the deposit and dissemination of scientific research documents, whether they are published or not. The documents may come from teaching and research institutions in France or abroad, or from public or private research centers.
L'archive ouverte pluridisciplinaire HAL, est destinée au dépôt et à la diffusion de documents scientifiques de niveau recherche, publiés ou non, émanant des établissements d'enseignement et de recherche français ou étrangers, des laboratoires publics ou privés. 


\title{
ADVANGES IN OPTIGALLY PUMPED He ${ }^{4}$ MAGNETOMETERS : RESONANGE AND NONRESONANGE TEGHNIQUES
}

\author{
By R. E. SLOGUM, \\ Texas Instruments Incorporated, Dallas, Texas (U.S.A.).
}

\begin{abstract}
Samples of optically pumped $2^{3} S_{1}$ helium atoms are currently studied in our laboratory for use in advanced magnetometer applications. The results of recent investigations of the effect of pumping beam orientation with respect to the observed magnetic field are presented in this paper. The orientation dependence of the amplitude and frequency of the magnetic resonance signal observed by optical techniques has been measured. These results are used to predict the effect of orientation on scalar field change measurements. The experiments are theoretically interpreted by taking into account the contribution of the individual magnetic resonances and the perturbation of magnetic states by real and virtual transitions. The orientation dependence of the optical pumping process is used to construct a nonresonance magnetometer (no magnetic resonance) suitable for measuring vector interplanetary and laboratory fields in the range $-200 \gamma \leqslant H \leqslant 200 \gamma$. Several new optical pumping components which have contribued to the advancement of helium magnetometers are discussed.
\end{abstract}

I. Introduction. - Optically pumped samples $[1,2]$ of $2^{3} S_{1} \mathrm{He}^{4}$ atoms are utilized as detecting elements in two types of magnetometers which can be characterized by their field detecting methods as either resonance or nonresonance devices. The resonance designation is applied to the various magnetic resonance techniques which employ optical detection of magnetic resonance and are used to observe changes in the scalar field. The nonresonance technique utilizes the orientation dependence of pumping beam absorption in an optically pumped gas to derive vector field information. It is the dependence of absorption on the angle between the beam and magnetic field rather than the Larmor frequency of the sample that is used to obtain vector field information in the helium magnetometers employed to measure interplanetary fields $[3,4]$. Because of the increasing interest in the measurement of low and vanishing magnetic fields in the laboratory as well as space, our work with the nonresonance technique will be reviewed.

The second part of this paper contains a description of a recent investigation of the orientation dependence of the resonance signal in optically pumped helium. The atoms of the sample are placed in a selective population distribution over the magnetic states by absorption of appropriately polarized 1.08 micron light composed of the $\mathrm{D}_{0}, \mathrm{D}_{1}$ and $\mathrm{D}_{2}$ lines. The orientation dependence of the optical pumping process in the level $2^{3} S_{1}$ and the orientation dependence of the frequency of the resonance signal have been measured. These results have been useful in understanding the orientation dependence of the frequency of optically pumped magnetometer sensors. The interpretation of these results has led to a better understanding of the optical pumping process in the $2^{3} S_{1}$ level and, in particular, to an understanding of the perturbation of the magnetic states by the pumping beam and the orientation dependence of these perturbations.

The optical pumping apparatus shown schematically in figure 1 is similar for both resonance and nonresonance experiments. The sample is contained in a cylindrical absorption cell and maintained at a weak level of $\mathrm{rf}$ discharge. The pumping radiation is obtained from an $\mathrm{rf}$ powered electrodeless helium lamp.

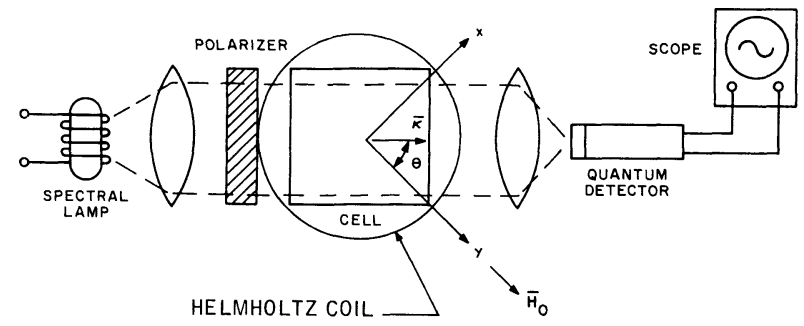

FIG. 1. - Optical pumping apparatus (schematic).

The light is circularly polarized and the absorption in the cell is observed by monitoring the transmitted beam. The Helmholtz coil, shown schematically, represents for the resonance case the means of applying a resonance field to the sample. In the nonresonance case, it is a means of changing the magnetic field direction at the sample to some angle $\theta$ with respect to the beam direction.

II. Nonresonance technique. - Turning first to the nonresonance technique, three measurements were performed to observe the absorption behavior of an optically pumped sample in regions of magnetic fields in the microgauss range. An optical pumping apparatus was placed in the region of homogeneous fields at the center of a large coil system operated by the Jet Propulsion Laboratory at a remote location north of Los Angeles, California. The three perpendicular coils, 10 meters in diameter, were used to reduce the field at the sample to the microgauss range. Despite lack of compensation of fluctuations of the earth's magnetic field, the field in the sample region could be effectively reduced to the microgauss range for short periods of time.

With the ambient field reduced to the microgauss range and the beam circularly polarized, a 5000 microgauss oscillating field was first applied along the beam axis. The field frequency was approximately $100 \mathrm{~Hz}$ and no change in absorption was observed. Since the relaxation time of the sample was approximately $10^{-4}$ seconds, the sample remains in an effective steady state at all times. The oscillating field direction was 
then changed to perpendicular the beam direction, and a change in absorption was observed as the field passed through the line width of the zero crossing resonance for $2^{3} S_{1}$ helium which is calculated to be approximately 1000 microgauss. These experiments were continued first in the room size magnetic shield of the Mobil Oil Co. and finally inside a shield consisting of 5 concentric mu-metal cylinders in our own laboratory.

The perpendicular and parallel oscillating fields were combined and phase shifted to generate a constant amplitude field at the sample which rotated in a plane containing the beam direction. With the ambient field $H_{0}$ reduced below one microgauss and a rotating field of amplitude $H^{\prime}=5000$ microgauss applied to the sample, the absorption signal appeared identical to that for a linear oscillating field perpendicular to the beam. The absorption signal $S(\omega t)$ in the presence of a rotating field can be expressed in terms of its harmonic components as :

$$
S(\omega t)=\sum_{n=1}^{\infty}\left(a_{n} \cos n \omega t+b_{n} \sin n \omega t\right) .
$$

With only a rotating field $H^{\prime}$ applied to the sample and the ambient component $H_{0}=0$, the signal is primarily at the second harmonic frequency. For values of $H_{0}<H^{\prime}$, however, components are introduced with term coefficients :

$$
\begin{gathered}
b_{1}=b_{3}=-a_{2} H_{\mathrm{y}} / 2 H^{\prime} \\
a_{1}=-a_{3}=a_{2} H_{\mathrm{x}} / 2 H^{\prime} .
\end{gathered}
$$

The signal is filtered to remove all but the fundamental component and phase sensitive demodulated to obtain voltages proportional to the field components parallel and perpendicular the beam direction in the plane of the sweep field. Each voltage is used to regulate the current in a Helmholtz coil surrounding the sample with axis parallel the corresponding field component. The field in each coil is servocontrolled to null the ambient field component and the magnitude of this component is determined by the instantaneous nulling current passing through the calibrated Helmholtz coils.

A three-axis device was constructed by adding a third perpendicular null coil and alternating the rotating sweep between two perpendicular planes containing the beam direction. This instrument was demonstrated for the National Aeronautics and Space Administration in 1962 and was selected for a series of measurements of interplanetary and near planet fields. Instruments of this type shown in figure 2 were utilized on the Mariner IV, Mars 1964 mission, and the Mariner V, Venus 1967 mission. The helium

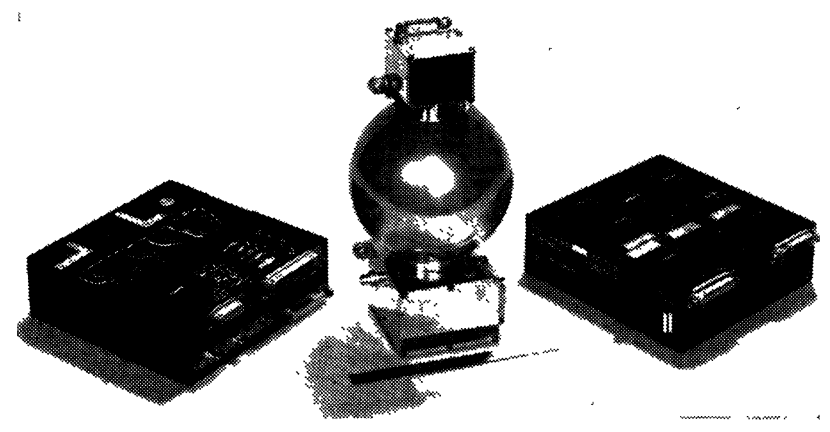

FIG. 2. - Nonresonance helium magnetometer. sample is enclosed inside a spherical form on which three perpendicular Helmholtz coils $(10 \mathrm{~cm}$ in diameter) are wound. Support electronics equipment is contained in the side modules. A basic noise threshold of 1 microgauss was achieved but not utilized since this is four times better resolution than the spacecraft telemetry. Both experiments were successful and the Mariner IV instrument was observed in normal operation in a post mission earth encounter after 1000 days of continuous operation in space. The range of the instrument is $\pm 200 \gamma$.

III. Resonance signal investigation. - I would like now to turn to a more recent investigation of the orientation dependence of the optically observed magnetic resonance signal in helium. Resonance magnetometers are frequently employed to detect scalar changes in the geomagnetic field at a fixed location or to detect magnetic anomalies from air or space borne platforms. In mobile applications in particular, it is desirable to eliminate if possible the dependence of the observed resonance frequency on the orientation of the optical pumping sensor with respect to the magnetic field. In pursuit of this objective, a basic study of the orientation dependence of magnetic resonance phenomena in optically pumped gases has been initiated.

The effect of orientation on Zeeman splitting is well known for paramagnetic crystals. In an analogous study of optically pumped helium, we have measured the orientation dependence of the amplitude and frequency of the optically observed resonance signal. The frequency variation is due to the perturbation of the magnetic states by the pumping beam investigated by Cohen-Tannoudji [5] and Schearer [6] for the case where the pumping beam and field are aligned. An optical pumping apparatus similar to that described in the nonresonance experiment was used for the orientation study. The sample consisted of 0.2 torr of research grade helium contained in a cylindrical glass cell which was maintained at a weak discharge level by $\mathrm{rf}$ excitation. The excitation field is applied by means of copper bands around the ends of the cylinder, one of which was connected to the oscillator and the other to ground. Care was taken to align the electrode axis with the beam and cell axis. This careful alignment allows the beam to effectively integrate out the gradients associated with the nonhomogeneous distribution of the rf discharge between the "hot" and ground electrodes. Gradients of up to $3 \mathrm{~Hz}$ per $\mathrm{cm}$ have been observed by cell scanning. The helium lamp which serves as the beam source is excited by a low-noise, solid state oscillator at $50 \mathrm{MHz}$. The transmitted beam is monitored with a high resistivity silicon detector with good spectral response at 1.08 micron.

The pumping apparatus pictured in figure 1 is mounted to a fixture which can be rotated through $360^{\circ}$. The rotation axis passes through the helium sample and is perpendicular to the plane containing the earth's magnetic field and the pumping beam. The resonance field is applied to the sample by means of a $20 \mathrm{~cm}$ diameter Helmholtz coil with its axis colinear with the axis of rotation. A large compass wheel is attached to the rotating fixture which allows the positioning of the apparatus at any angle $\theta$ with respect to the field to within $\pm 1 / 8$ degree.

An electromagnetically quiet, low gradient region 
was desirable for this experiment. The location selected was the Dallas Magnetic Observatory operated by the U.S. Coast and Geodetic Survey where nonmagnetic buildings are maintained for the use of magnetics experiments. The optical pumping apparatus, rotating fixture, support electronics and a portion of the reference helium magnetometer were located in a small nonmagnetic building. All items in this building were constructed of nonmagnetic components. The remaining support and measuring electronics were located in a second nonmagnetic building 75 meters away.

The experimental techniques used to observe the resonance signal are outlined in figure 3 . In the top illustration for the signal amplitude measurement, the resonance oscillator supplying the resonance rf field to

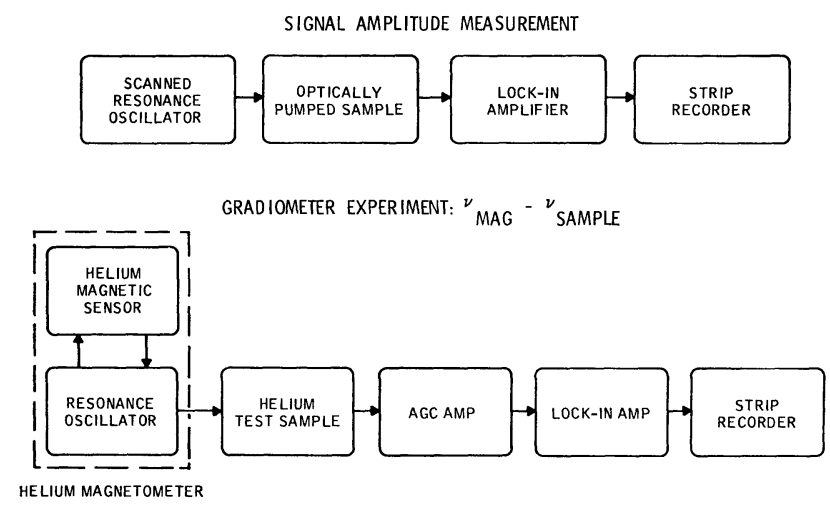

FIG. 3. - Outline of experimental techniques.

the sample is scanned slowly through the resonance line. The oscillator is simultaneously frequency modulated about the center frequency with a $500 \mathrm{~Hz}$ square wave. The af modulation amplitude is adjusted to a value much greater than the $4.6 \mathrm{kHz}$ linewidth of the sample. The beam detector output is directed into a lock-in amplifier for demodulation, and the amplifier output is continuously recorded. The relative amplitude of the signal is then equal to the difference of the amplifier output for no resonance field and the peak point reached for either phase of a complete line scan.

The shift in the frequency of the resonance signal is observed by comparing the sample frequency $v_{s}$ with that of a reference helium sample $\nu_{m}$ as indicated at the bottom of this figure. The reference sample is the optically pumped helium in the reference helium magnetometer sensor. The resonance oscillator of the magnetometer tracks the resonance frequency $v_{m}$ of the sensor sample and this oscillator output is frequency modulated with a $500 \mathrm{~Hz}$ square wave about the center frequency and used to supply a resonance field to the test sample. The signal generated on the transmitted beam is detected and fed to a lock-in amplifier and recorded. The voltage out of the lock-in amplifier is proportional to $\Delta v$ and is calibrated in Hz. An automatic gain control amplifier utilizing a second harmonic pulse maintains the calibration of the output voltage even though the signal may vary in amplitude. The voltage is calibrated by electronically shifting the center frequency corresponding to $\nu_{m}$ while $\nu_{s}$ is held constant and observing the resulting voltage change. This arrangement has the additional feature of a gradiometer in eliminating geomagnetic fluctuations from the measurement of the frequency difference.
The helium reference and test samples are located a meter apart, and the resonance frequencies are related to the field value by the same proportionality constant. The frequency difference is then independent of the magnitude of the ambient magnetic field.

The resonance signal amplitude shown in figure 4 was measured over the range from $0^{\circ}$ to $180^{\circ}$ to obtain

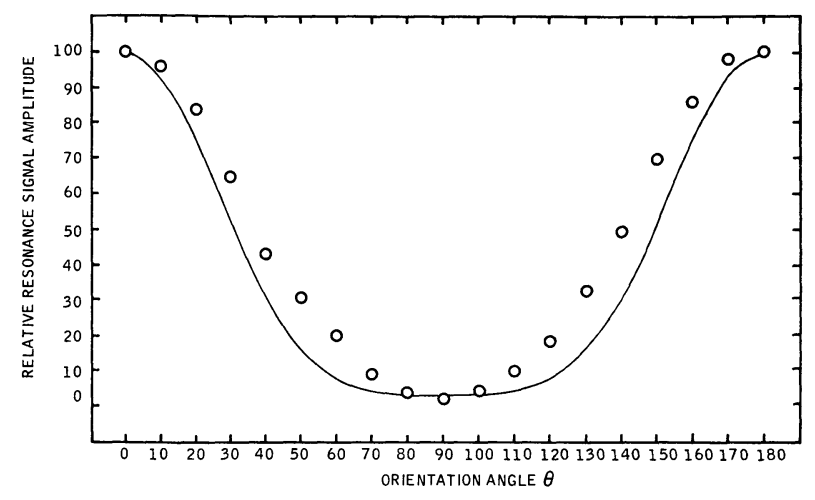

FIG. 4. - Measured and calculated resonance signal amplitude dependence on orientation.

the relative values indicated by the open circles. Maximum amplitude is obtained when the beam and field are coaxial, and a value less than $4 \%$ of maximum is measured for $\theta=90^{\circ}$. The orientation dependent population numbers were calculated for the $2^{3} S_{1}$ states and used to derive an expression for the resonance signal observed for each of the resonances of the optically pumped level. The two signals were combined to obtain the theoretical signal variation indicated by the solid line. The orientation lag of the theoretical curve by the experimental points as the beam and field diverge is due to experimental deviations from the theoretical assumptions of collimated light and homogeneous, optically thin samples. An estimate of the lag angle introduced by the apparatus was obtained by comparing the angular displacement of the experimental curve with the theoretical curve and have been useful in the analysis of the frequency variation data.

In figure 5 is shown the measured variation of the

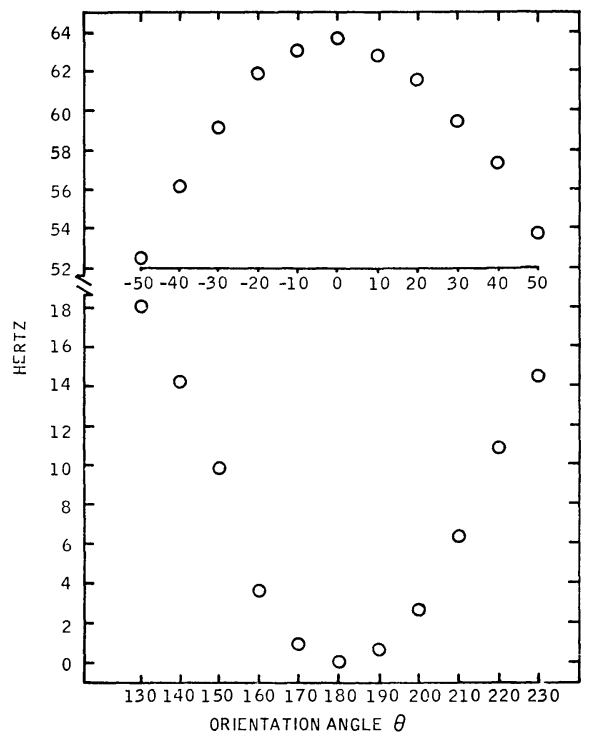

FIG. 5. - Measured and calculated variation of the resonance signal frequency with orientation. 
resonance signal frequency with angle. The ability of the agc amplifier to maintain the calibration for reduced signal amplitude limited the measurements to angle of divergence of the beam from the field axis of less than $50^{\circ}$. The $180^{\circ}$ position was selected as the reference level and shifts were measured from this value. The shift between the $0^{\circ}$ and $180^{\circ}$ position was measured to within $\pm 0.7 \mathrm{~Hz}$. The frequency changes with respect to $0^{\circ}$ and $180^{\circ}$ values were measured to within $\pm 0.15 \mathrm{~Hz}$ giving good resolution to the curves of frequency variation with angle.

Each point on the lower curve is measured at an orientation $180^{\circ}$ from the measured point directly above it. Each pair of vertically matched points represents an effective field reversal for the sample. Upon comparing the shapes of the frequency variation curves, it is apparent that the orientation dependence is nonsymmetric with respect to the field reversal. The theoretical analysis of the off-resonant light shift indicates that this shift is symmetrical with respect to field reversal. It should be possible to determine the magnitude of the virtual transition shift by measuring one-half the separation between any vertical pair of points.

The magnitude of the shift introduced by the offresonant light shift has been calculated using the data shown in figure 5 . The magnitude is observed to decrease with increasing divergence of the beam and field as shown by the open circles in figure 6 . This data has been corrected for orientation and experimental offset and can be compared with the theoretical predictions of the shift variation indicated by the solid line. This agreement between the observed and calculated values substantiates the explanation that the variation in frequency which is symmetric with respect to field reversal is due to the off-resonant radiation. There remains, however, a variation of the frequency

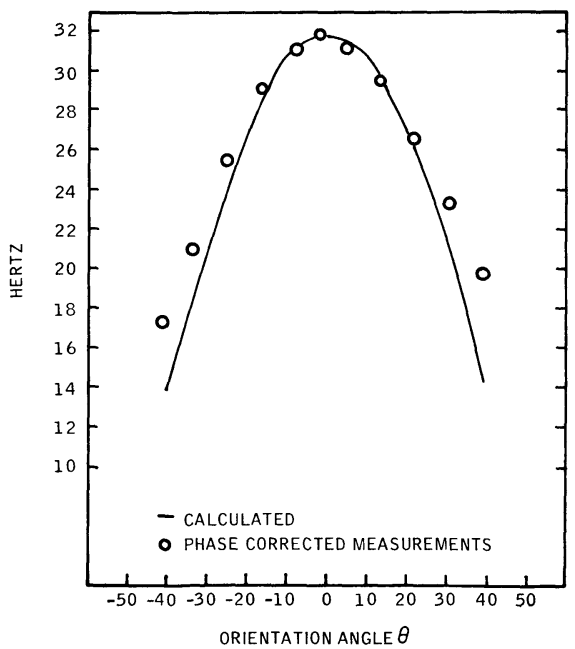

FIG. 6. - Orientation dependence of magnitude of nonresonant light shift.

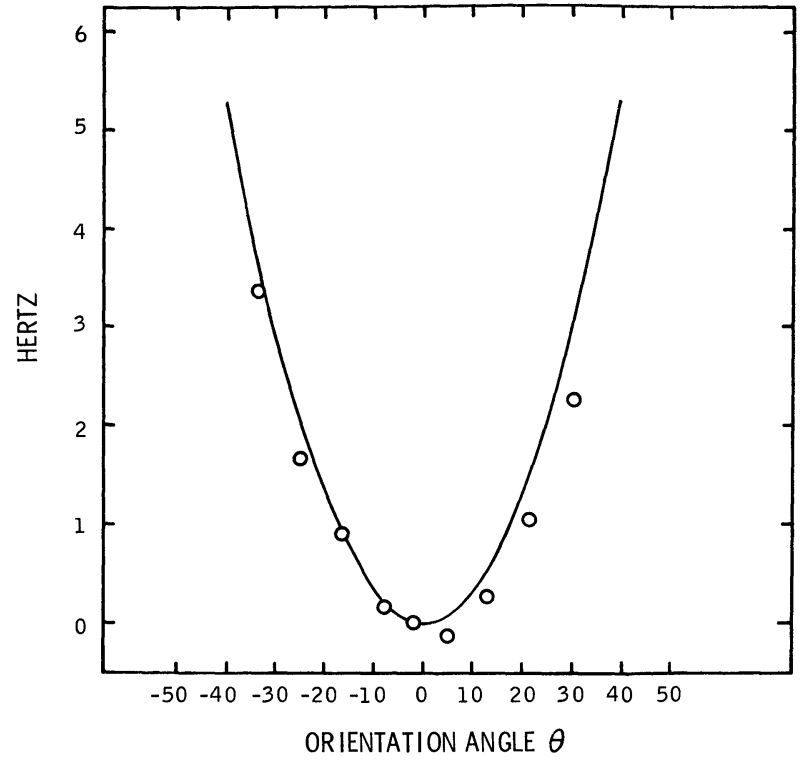

FIG. 7. - Orientation dependence of coherence transfer shift.

of the resonance signal with angle which is independent of field reversal.

This shift is displayed by the open circles in figure 7 and represents the variation in shift magnitude with the effects of off-resonant radiation removed. To explain this effect, it was observed that the coherence transfer shift contributed by real transitions produced a frequency shift of approximately $60 \mathrm{~Hz}$ for the $0^{\circ}$ and $180^{\circ}$ position. An expression was developed for the orientation dependence of the coherence transfer case which predicts the values indicated by the solid line in figure 7. The agreement of the observed and calculated values indicate that the portion of the frequency shift which is independent of field reversal is due to the orientation dependence of the coherence transfer shift.

In summary the variation of the frequency of the optically observed resonance signal with orientation angle is found to be nonsymmetrical with respect to field reversal. This nonsymmetry is explained by the fact that two types of shifts introduced by the pumping beam are orientation dependent. The variation with angle of the nonresonant light shift is symmetric with respect to field reversal and decreases in magnitude with the angle of divergence. The coherence transfer shift is found to be independent of field reversal and increases with the divergence of the beam from the field axis. It can be concluded, therefore, that for optical pumping magnetometer sensors, the orientation dependence of the observed frequency cannot be eliminated solely by compensating by dual beam techniques the virtual light shifts, but effective means must be devised to suppress the coherence transfer shift as well.

\section{REFERENCES}

[1] Colegrove (F. D.) and Franken (P. A.), Phys. Rev., 1960, 119, 683.

[2] SchEARER (L. D.), Advances in Quantum Electronics (Columbia University Press, New York, 1961).

[3] SLOCum (R. E.) and REILIY (F. N.), I.E.E.E. Trans. on Nuclear Science, 1963, NS-10, 165.
[4] Conners (B. V.), I.E.E.E. Trans. on Magnetic, 1968, MAG-4, 391.

[5] Cohen-Tannoudji (C.), Ann. Phys., 1962, 7, 423 ; 1962, 7, 469.

[6] Schearer (L. D.), Phys. Rev., 1962, 127, 512. 